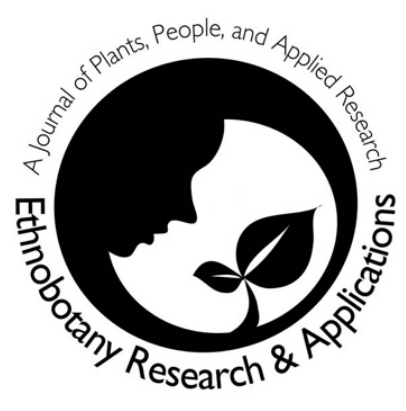

Facteurs influençant l'efficacité des recettes galactogènes chez les vaches Borgou élevées en milieu réel au Bénin Factors influencing the effectiveness of galactogenic recipes in Borgou cows reared in a real environment in Benin

Agani Zénabou, Boko K. Cyrille, Adenile Dourossimi Adam, Akouedegni C. Guénole, Mensah Guy Apollinaire, Dossou Joseph and Babatounde Séverin

\section{Notes on Ethnobotany}

\section{Résumé}

Objectif: La médecine ethno-vétérinaire fait partie intégrante du patrimoine culturel associé à la médecine traditionnelle en Afrique, où les plantes et les produits naturels traitent les maux dont souffrent les animaux. De nombreuses plantes sont utilisées pour améliorer la performance laitière des vaches locales. L'étude évalue les facteurs influençant l'efficacité des recettes galactogènes administrées aux vaches Borgou élevées en milieu réel au Bénin.

Méthodologie: Une enquête a été réalisée en pleine saison pluvieuse (avril à septembre 2019), à l'aide d'un "focus group " afin de classer les plantes impliquées dans les recettes galactogènes. Les résultats obtenus ont été soumis à une Analyse Factorielle des Correspondances (AFC) suivie d'une régression multiple effectuée sur des données qualitatives notamment, les organes des plantes utilisées, les modes d'utilisation, les additifs utilisés et la voix d'administration de la recette.

Correspondence
Agani Zénabou ${ }^{1 *}$, Boko K. Cyrille ${ }^{4}$, Adenile
Dourossimi Adam ${ }^{3}$, Akouedegni C. Guénole ${ }^{3}$,
Mensah Guy Apollinaire ${ }^{2}$, Dossou Joseph ${ }^{5}$ and
Babatounde Séverin'
'Laboratoire de Zootechnies, Faculté des Sciences,
Université d'Abomey-Calavi, 01 BP 526, Cotonou,
zenabagani@gmail.com
${ }^{2}$ Institut National de Recherche Agricole du Benin
(INRAB) mensahga@gmail.com
${ }^{3}$ Laboratoire d'Ethnopharmacologie et de Santé
Animales FSA / UAC
${ }^{4}$ Unité de recherche sur les maladies transmissibles,
EPAC / UAC
${ }^{5}$ Laboratoire de bio-ingénierie des procédés
alimentaires LaBioPA
${ }^{*}$ Corresponding Author: zenabagani@gmail.com
Ethnobotany Research \& Applications
21:08 (2021)


Résultats: Arachis hypogaea (L.), Euphorbia balsamifera Aiton et Gardenia aqualla Stapf \& Hutch sont les espèces les plus efficaces pour améliorer la performance laitière des vaches locales. Des 44 familles de plantes galactogènes inventoriées, dix (Fabaceae Apocynaceae, Euphorbiaceae, Moraceae, Meliaceae, Poaceae, Sapotaceae, Asteraceae, Lamiaceae et Rubiaceae), ont influencé significativement $(P<0,05)$ l'efficacité des recettes. Les organes des plantes les plus utilisées ont été les racines et les graines. La potasse est le seul additif qui impacte positivement l'efficacité des préparations. Parmi les voix d'administration identifiées, la voie orale apparaît plus efficace.

Conclusion: L'étude montre que les agro-éleveurs ont une parfaite connaissance des différents facteurs qui influencent l'efficacité des recettes galactogènes. Toutefois, certaines espèces végétales impliquées se raréfient à cause du développement des champs de cultures au détriment du couvert forestier. La plupart des recettes efficaces font intervenir les racines. Ceci pose de réels problèmes pour l'écologie du milieu et le développement des espèces impliquées. Des expérimentations sont en cours afin de confirmer les résultats issus des enquêtes.

Mots clés: Connaissance endogène; plantes galactogènes; influence; production; lait; organes de plantes; Bénin.

\begin{abstract}
Background: Ethnoveterinary medicine is an integral part of the cultural heritage associated with traditional medicine in Africa, where plants and natural products treat the ailments suffered by animals. Many plants are used to improve the dairy performance of local cows. The study assesses factors that influencing the effectiveness of galactogenic recipes administered to Borgou cows raised in real conditions in Benin.

Methodology: A survey was carried out in the middle of the rainy season (April to September 2019), using a "focus group" to classify the plants involved in galactogenic recipes. The results obtained were subjected to a Factorial Correspondence Analysis (AFC) followed by a multiple regression carried out on qualitative data in particular, the organs of the plants used, the modes of use, the additives used and the administration route of recipe.
\end{abstract}

Results: The Factorial Correspondence Analysis (CFA) carried out on the methods of choosing the plants given relative to the zones showed that the correlation between these two variables was explained at $66.69 \%$ by the first two axes. Therefore, the involvement of species in revenue depends on their availability in the study area. Indeed, the correspondences resulting from the projection of the different plants used in galactogenic preparations and the different assessment methods of agrobreeders (Efficient, moderately efficient, and fair) in the AFC axis systems (Figure 1) showed that in the communes of Gogounou and Kalalé, Arachis hypogaea (L.) and Euphorbia balsamifera Aiton were the most effective species. On the other hand, in Djidja, the most efficient plant was Gardenia aqualla Stapf \& Hutch.

For each species, the results of the multiple regressions carried out with the organs involved in the preparation of galactogenic recipes are shown in Table 1. The organs exhibiting a positive coefficient and a probability less than 0.05 are those which positively influenced milk production. Note that for the species presented in the above table, one or more organs can be used in recipes presumed to be galactogenic by traditional breeders. But the effectiveness of the recipe depends on the type involved in its constitution. It is interesting to note that in certain species: Aganope stuhlmannii (Taub.) Adema, Vitellaria paradoxa C.F.Gaertn, Calotropis procera subsp. hamiltonii (Wight) Ali, Khaya senegalensis (Desv.) A.Juss, Vigna unguiculata (L.) Walp, Gardenia aqualla Stapf \& Hutch, Daniellia oliveri (Rolfe) Clapier. Et Dalziel, Saba comorensis (Bojer ex A.DC.) Pichon and Vitellaria paradoxa C.F.Gaertn, several organs positively impacted milk production. However, for the same recipe, the association of several organs in its constitution has a depressive effect on milk production. Apart from the number of organs involved in the constitution of the recipes, the method of preparation associated with each plant species impacted the milk production of the cows. Out of the thirteen species (19) which had significant effects with milk production, the results of Table 2 indicate that the modes of use were successively ranked as follows (Table 2): Maceration> decoction> powder> infusion were cited. Among the species which have undergone maceration: Budgunnia madagascariensis (Desv.) JHKirkbr. \& Wiersema, Arachis hypogaea (L.), Euphorbia balsamifera Aiton and Curcubita maxima (L.) have been mentioned. Species such as: Gardenia aqualla Stapf \& Hutch, Sorghum bicolor (L.) Moench and Vittelaria paradoxa C.F. Gaertn could only be effective if used as a decoction. The effectiveness of Vigna unguiculata (L.) Walp on milk production could only be obtained by trituration for one hour. As for Saba comorensis (Bojer ex A.DC.) Pichon, the method of use was only very effective by infusion $(P<0.001)$.

Among the ingredients involved in the recipes, only salt and potash appeared to be significant $(P<0.05)$ on the milk production of cows (Table 2). In addition, the results of the work indicated that the 
administration of oral preparations positively influenced milk production $(P<0.05)$.

Conclusion: The study shows that agro-pastoralists have a perfect knowledge of the different factors that influence the effectiveness of galactogenic recipes. However, some plant species involved are becoming rare because of the development of crop fields to the detriment of forest cover. Most effective recipes involve the roots. This poses real problems for the ecology of the environment and the development of the species involved. Experiments are underway to confirm the results of the surveys.

Keywords: Indigenous knowledge; Galactogenic; plants; affecting; production; milk; organs; Bénin.

\section{Introduction}

Les connaissances endogènes sur l'utilisation des plantes médicinales pour traiter les animaux et les hommes remontent à des milliers d'années. La documentation sur les savoirs endogènes a acquis une importance considérable dans le monde, en particulier avec la ratification du protocole de Nagoya afin de préserver le patrimoine culturel (Aubertin, 2018; Ayeni et Basiri, 2018). La convention sur la biodiversité signifie préserver et maintenir les connaissances, les innovations et les pratiques des communautés autochtones incarnant le mode de vie traditionnel pertinent pour la conservation et l'utilisation durable de la biodiversité (Salgotra et al., 2018). La connaissance est normalement transmise de génération en génération et de bouche à oreille (Hussain et al., 2018), dans les histoires, les poèmes, les proverbes et les chansons. Cependant, la transmission des savoirs traditionnels par les méthodes orales n'est pas fiable et le recours à la documentation est nécessaire (Mullah et al., 2013). Les plantes ethno- vétérinaires ont la capacité de lutter contre différents types de maladies animales (Bullitta et al., 2018) et présentent plusieurs avantages par rapport aux médicaments synthétiques. Les plantes ethno-vétérinaires gagnent maintenant en popularité dans les pays développés et en développement, car ils sont facilement disponibles et peuvent être récupérées dans la nature.

L'élevage pastoral joue un rôle prépondérant dans l'économie des pays de l'Afrique tropicale (Lesse et al., 2015). Au Bénin, le cheptel national est estimé à 4,6 millions de têtes dont 2,4 millions de bovins en 2019 (DE, 2019) avec une production du lait estimée à 37 millions de tonne de lait de vache. Dans ce pays, le lait joue un rôle majeur dans l'économie rurale en fournissant des revenus et des emplois aux éleveurs et aux femmes de la société agro-pastorale.
Malgré les efforts consentis par le programme national de développement des filières lait et viande visant à améliorer la productivité des animaux d'élevage au Bénin, la production en lait des bovins demeure faible. La principale contrainte à lever est le faible niveau de productivité laitière de la plupart des races bovines locales. Les éleveurs n'arrivent pas à adopter les nouvelles stratégies mises en œuvre par l'état béninois. Pour cela, ils sont obligés de se référer aux méthodes traditionnelles plus faciles, et accessibles à moindre coût, notamment, l'utilisation des recettes galactogènes. Récemment, les travaux de (Agani et al., 2019) ont confirmé I'utilisation des préparations galactogènes pour améliorer la production du lait des vaches locales. Toutefois, ces travaux ne précisent pas l'influence des espèces végétales, les parties ou organes des plantes utilisées, les additifs associés aux recettes et même la voix d'administration sur l'efficacité des préparations administrées aux vaches locales. L'objectif de ce travail est d'évaluer les facteurs influençant l'efficacité des recettes galactogènes sur les vaches Borgou élevées en milieu réel au Bénin.

\section{Matériaux et Méthodes}

\section{Milieu d'étude}

L'étude a été menée au Nord du Bénin dans les communes de Gogounou, de Kalalé et au Sud dans la commune de Djidja. Ces communes ont la plus forte concentration de l'effectif du bétail et de grand marché à bétail. De plus, elles disposent d'une diversité écologique qui leur permet d'utiliser une variété d'espèce végétales entrant dans la préparation des recettes galactogènes. La Direction de L'Elevage (D.E., 2018), a souligné que la commune de Kalalé occupe la première place en effectif bovin dans les départements du Borgou et de l'Alibori (Nord-Est du Bénin). Elle est située entre $3^{\circ}$ $22^{\prime} 54^{\prime \prime}$ de longitude Est et $10^{\circ} 17^{\prime} 24^{\prime \prime}$ de latitude Nord. Cette commune est située en zone soudanienne, avec une alternance de pluies (avril à octobre) et de saison sèche (Novembre à Mars) (Salifou et al., 2017). La pluviométrie annuelle varie entre 845 et $1322 \mathrm{~mm}$, et suit une distribution unimodale. Le système d'utilisation de la terre est basé sur les cultures du sorgho et de l'igname, avec une forte concentration en des champs de maiis et de coton. Par contre, la commune de Gogounou, a une superficie de $20930 \mathrm{~km}^{2}$, et est comprise entre les parallèles. $10^{\circ} 29^{\prime} 34^{\prime \prime}$ et $11^{\circ} 45^{\prime} 25^{\prime \prime}$ de latitude Nord et les méridiens $01^{\circ} 42^{\prime} 54^{\prime \prime}$ et $3^{\circ} 43^{\prime} 51^{\prime \prime}$ de longitude Est. Par ailleurs, encore appelé le grenier du Zou, la commune de Djidja est comprise entre les parallèles $7^{\circ} 10^{\prime}$ et $7^{\circ} 40^{\prime}$ de latitude Nord, et les méridiens $1^{\circ} 04^{\prime}$ et $2^{\circ} 10^{\prime}$ de longitude Est et caractérisée par un climat de type subéquatorial tendant vers le soudano-guinéen dans les parties septentrionales avec deux saisons pluvieuses. 


\section{Méthode d'enquête}

L'enquête a été réalisée en pleine saison pluvieuse (avril à septembre 2019), auprès de 565 agroéleveurs de la zone soudano-guinéen au Sud et au Nord du Bénin au moyen d'interviews directes. Les enquêtés ont été choisis avec l'aide des responsables de l'Agence Territoriale du Développement Agricole (ATDA) et des Chefs d'Arrondissement suivant les critères définis par (Eynden et Damme, 1993) à savoir: disponibilité et habitude à travailler avec les agents d'élevage, la célébrité dans la pratique de la médecine traditionnelle; la réputation ou la popularité du village en médecine traditionnelle; l'usage exclusif ou majoritaire des produits végétaux ou naturels dans le processus de guérison. A ces critères, y est ajouté un autre défini par (Dassou et al., 2020) qui est la concentration élevée d'animaux pendant la période de l'enquête. Toute personne d'un intervalle d'âge de 25 ans à 80 ans ayant une connaissance sur les pratiques endogènes utilisées pour induire ou améliorer la production du lait ont été prises en compte. Sur les 284 recettes galactogènes recensées, une classification numérique a été faite sur celles ayant été citées par au moins $20 \%$ des enquêtés. Ensuite, un focus group a été effectué dans chaque zone agro-écologique afin de classer les meilleures espèces végétales impliquées dans les recettes. A cet effet des scores ont été attribués par les répondants sur la base d'une grille d'appréciation à quatre critères $(3=$ espèce fortement utilisée; 2 = espèce moyennement utilisée; 1 = espèce faiblement utilisée; 0 = espèce sans usage) (Dossou et al., 2012). En préliminaires, les informations sur les différentes espèces entrant dans la constitution des recettes, les parties des organes et les ingrédients ajoutés à ces recettes, le mode de préparation, la forme d'administration associée à chaque espèce ainsi que la voix d'administration ont été collectées.

\section{Analyses statistiques}

Les espèces végétales citées lors des entretiens ont été recherchées avec l'aide des enquêtés. Des images et des échantillons ont été pris sur celles disponibles au cours de la période d'enquête. Par la suite, des échantillons des espèces ont été constitués sous forme d'herbiers pour une identification ultérieure à l'aide de la Flore Analytique du Bénin et la liste des espèces a été établie suivant la nomenclature botanique utilisée par (Akoègninou et al., 2006) puis confirmée sur le site http://www.plantlist.

Pour établir une relation entre l'utilisation efficace d'une espèce végétale et les zones d'étude, une Analyse Factorielle des Correspondances (AFC) a été réalisée avec le logiciel $R$ ( $R$ Core Team, 2019) et le niveau de significativité des tests statistiques a été fixé à $5 \%$. Seules les variables significatives $($ Prob $<0,05)$ à ce seuil ont été interprétées.

Les données essentiellement qualitatives relatives aux différents organes des plantes utilisées, à leurs modes d'utilisation, aux différents additifs utilisés et à la voix d'administration de la recette ont été soumises à une Classification Hiérarchique sur Analyses en Composantes Principales (ACP) afin d'identifier les différentes recettes pour l'amélioration de la production laitière. L'ACP réalisée avec le package FactoMineR (Sébastien et al., 2008), a consisté en une Analyse des Correspondances Multiples (ACM) réalisée sur les variables qualitatives binaires suivi d'une Classification Hiérarchique Ascendante (CHA) sur les composantes principales issues de l'ACM. Ensuite, une régression multiple a été effectuée afin de déceler les facteurs influençant significativement le gain laitier. Les variables impliquées dans le modèle ont été choisies par la méthode de sélection pas à pas (fonction step de R).

\section{Résultats}

Relation entre la perception des éleveurs sur les espèces végétales constituants les préparations galactogènes et la zone d'étude

L'Analyse Factorielle des Correspondances (AFC) réalisée sur les modalités de choix des plantes données relatives aux zones a montré que la corrélation entre ces deux variables était expliquée à 66.69 \% (Figure 1) par les deux premiers axes. Par conséquent, l'implication des espèces dans les recettes est fonction de leur disponibilité dans la zone d'étude.

En effet, les correspondances issues de la projection des différentes plantes entrant dans les préparations galactogènes et des différentes modalités d'appréciation des agro-éleveurs (Efficace, moyennement efficace, et passable) dans les systèmes d'axes de l'A.F.C. (Figure 1) ont montré que dans les communes de Gogounou et Kalalé, Arachis hypogaea L. et Euphorbia balsamifera Aiton ont été les espèces les plus efficaces. Par contre, à Djidja, la plante la plus efficace a été Gardenia aqualla Stapf \& Hutch., Vigna unguiculata (L.) Walp, Sorghum bicolor (L.) Moench, Budgunnia madagascariensis (Desv.) J.H. Kirkbr. \& Wiersema, et Saba comorensis (Bojer ex A. DC.) Pichon apparaissaient moyennement dans les communes de Kalalé et Gogounou. II en a été de même pour Arachis hypogaea L. et Afzelia africana Pers. dans la commune de Djidja. 


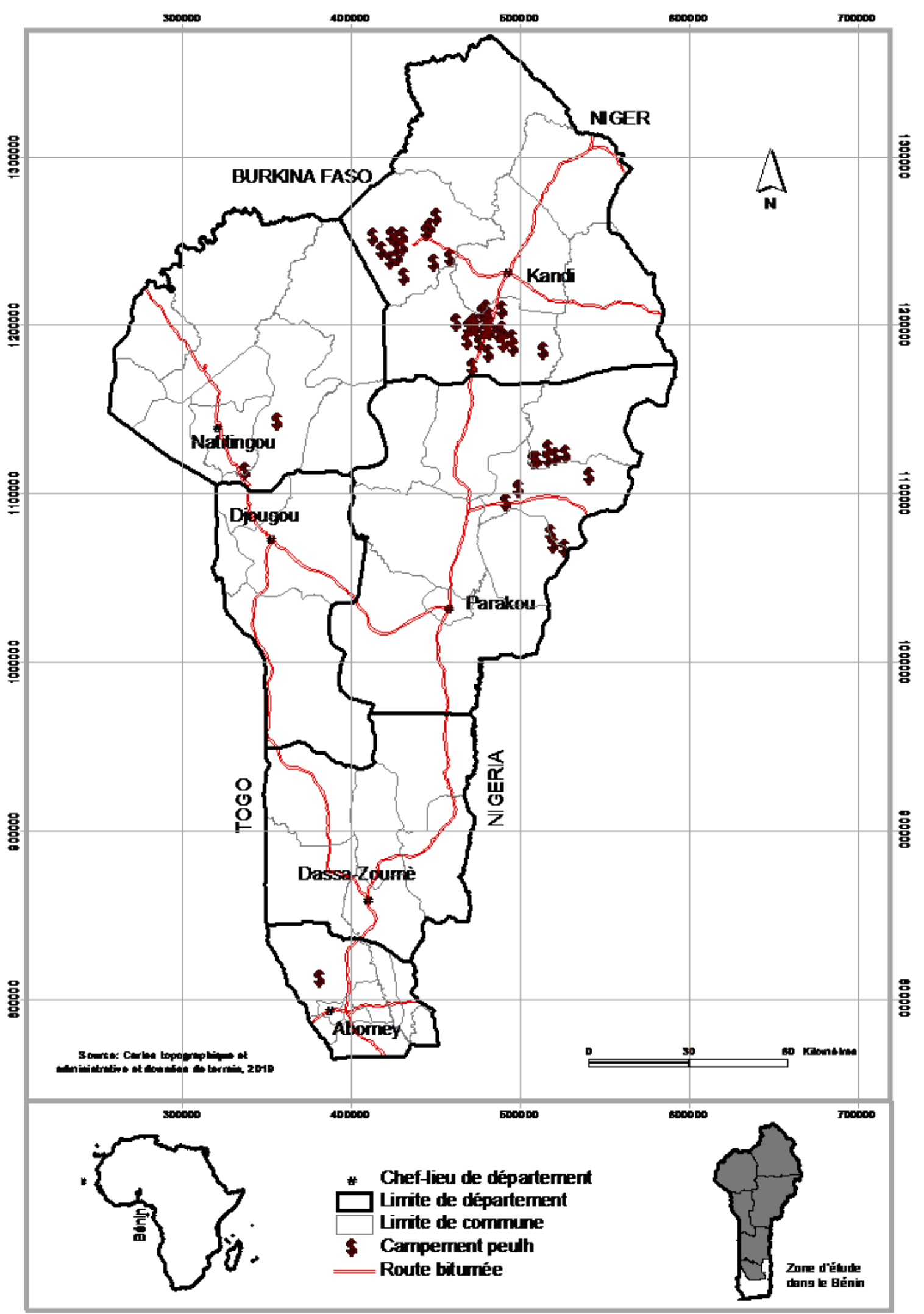

Figure 1. Cartographie de la zone d'étude Figure 1. Study area 
Facteurs influençant l'efficacité des recettes galactogènes

Pour chaque espèce, les résultats des régressions multiples effectuées avec les organes impliqués dans la préparation des recettes galactogènes sont consignés dans le Tableau 1 . Les organes présentant un coefficient positif et une probabilité inférieure à 0,05 sont ceux qui influençaient positivement la production laitière. Remarquons que pour les espèces présentées dans le tableau sus indiqué, un ou plusieurs organes peuvent être utilisés dans les recettes présumées galactogènes par les éleveurs traditionnels. Mais, l'efficacité de la recette est fonction du type et du nombre d'organes impliqués dans sa constitution. II est intéressant de constater que chez certaines espèces: Aganope stuhlmannii (DC.) Guill. \& Perr., Vitellaria paradoxa C.F. Gaertn, Calotropis procera subsp. hamiltonii (Wight) Ali, Khaya senegalensis (Desv.) A. Juss, Vigna unguiculata (L.) Walp, Gardenia aqualla Stapf
\& Hutch, Daniellia oliveri (Rolfe) Clapier.et Dalziel, Saba comorensis (Bojer ex A. DC.) Pichon et Vitellaria paradoxa C.F. Gaertn., plusieurs organes impactaient positivement la production laitière. Toutefois, pour une même recette, l'association de plusieurs organes dans sa constitution a un effet dépressif sur la production laitière. Avec l'espèce Aganope stuhlmannii (DC.) Guill. \& Perr, la production laitière est influencée quel que soit l'organe impliqué dans la recette. Par contre, pour les espèces telles que: Saba comorensis (Bojer ex A. DC.) Pichon, Gardenia aqualla Stapf \& Hutch, Ocimum basilicum L. et Bubgunnia madagascariensis (Desv.) J.H. Kirkbr. \& Wiersema, la production laitière n'est influencée que si les racines de ces plantes sont ajoutées aux recettes. En ce qui concerne Manihot esculenta Crantz et Raphionacme brownii Scott-Elliot, seul l'implication des tubercules et racines tubérisées a permis une amélioration de la production laitière (Figure 2).

Tableau 1. Les organes de plantes influençant la production du lait $\left(R^{2}=90,1 \%\right.$; Prob.Fisher $\left.(76 ; 466)<0,001\right)$ Table 1. Plant organs influencing milk production $\left(R^{2}=90.1 \%\right.$; Prob.Fisher $\left.(76 ; 466)<0.001\right)$

\begin{tabular}{|c|c|c|c|c|}
\hline Catégorie & Famille & Organe utilisé & Coef & Coef.ET \\
\hline Adansonia digitata L. ${ }^{(v)}$ & Malvaceae & Ecorce & 0,355 & 0,213 \\
\hline Afzelia africana Pers. ${ }^{(v)}$ & Fabaceae & Feuille & $0,129^{*}$ & 0,051 \\
\hline \multirow[t]{3}{*}{ Aganope stuhlmannii (Taub.) Adema } & \multirow[t]{3}{*}{ Fabaceae } & Ecorce & $1,323^{\star *}$ & 0,260 \\
\hline & & Feuille & $0,990^{* * *}$ & 0,215 \\
\hline & & Tige & $0,923^{\star *}$ & 0,300 \\
\hline \multirow[t]{2}{*}{ Arachis hypogaea L. } & \multirow[t]{2}{*}{ Fabaceae } & Graine & $0,921^{* *}$ & 0,034 \\
\hline & & Fane & 0,134 & 0,011 \\
\hline Allium sativum $\mathrm{L}$. & Amaryllidaceae & Feuille & 0,405 & 0,216 \\
\hline Balanites aegyptiaca (L.) Delile ${ }^{(v)}$ & Zygophyllaceae & Ecorce & 0,099 & 0,060 \\
\hline Bambusa vulgaris Schrad. & Poaceae & Feuille & 0,183 & 0,119 \\
\hline Bobgunnia madagascariensis (Desv.) ${ }^{(v)}$ J.H. Kirkbr. \& & Fabaceae & Ecorce & 0,165 & 0,092 \\
\hline Wiersema & & Racine & $0,130^{* *}$ & 0,029 \\
\hline Carica papaya L. (v) & Caricaceae & Graines & 0,443 & 0,299 \\
\hline Combretum adenogonium Steud. ex A. Rich. & Combretaceae & Feuille & 0,298 & 0,213 \\
\hline \multirow[t]{4}{*}{ Daniellia oliveri (Rolfe) Clapier. \& Dalziel } & \multirow[t]{4}{*}{ Fabaceae } & Ecorce & $0,180^{*}$ & 0,074 \\
\hline & & Feuille & 0,158 & 0,095 \\
\hline & & Racine & 0,212 & 0,151 \\
\hline & & Tige & $0,503^{*}$ & 0,218 \\
\hline \multirow[t]{3}{*}{ Saba comorensis (Bojer ex A. DC.) Pichon ${ }^{(v)}$} & \multirow[t]{3}{*}{ Apocynaceae } & Racine & $0,340^{* *}$ & 0,213 \\
\hline & & Tige & 0,013 & 0,213 \\
\hline & & Fruit & 0,117 & 0,297 \\
\hline \multirow[t]{3}{*}{ Calotropis procera subsp. hamiltonii (Wight) Ali(v) } & \multirow[t]{3}{*}{ Apocynaceae } & Feuille & $0,233^{*}$ & 0,155 \\
\hline & & Tige feuille & $0,124^{* *}$ & 0,023 \\
\hline & & Racine & 0,607 & 0,412 \\
\hline
\end{tabular}




\begin{tabular}{|c|c|c|c|c|}
\hline \multirow[t]{2}{*}{ Euphorbia balsamifera Aiton (v) } & \multirow[t]{2}{*}{ Euphorbiaceae } & Feuille & $0,119^{* *}$ & 0,032 \\
\hline & & Plante entière & $-0,957^{* *}$ & 0,299 \\
\hline \multirow[t]{2}{*}{ Ficus glumosa Delile } & \multirow[t]{2}{*}{ Moraceae } & Ecorce & $0,675^{\star *}$ & 0,175 \\
\hline & & Racine & $0,463^{* *}$ & 0,149 \\
\hline \multirow[t]{3}{*}{ Ficus vallis-choudae Delile } & \multirow[t]{3}{*}{ Moraceae } & Ecorce & $0,614^{*}$ & 0,320 \\
\hline & & Racine & 0,194 & 0,149 \\
\hline & & Fruit & 0,253 & 0,175 \\
\hline \multirow[t]{2}{*}{ Gardenia aqualla Stapf \& Hutch. ${ }^{(v)}$} & \multirow[t]{2}{*}{ Rubiaceae } & Racine & $0,594^{*}$ & 0,078 \\
\hline & & Fruit & 0,124 & 0,145 \\
\hline Guiera glandulosa Sm. & Combretaceae & Racine & 0,298 & 0,213 \\
\hline \multirow[t]{3}{*}{ Khaya senegalensis (Desv.) A. Juss. ${ }^{(v)}$} & \multirow[t]{3}{*}{ Meliaceae } & Feuille & $0,547^{*}$ & 0,070 \\
\hline & & Ecorce & $0,975^{* *}$ & 0,149 \\
\hline & & Racine & 0,213 & 0,034 \\
\hline \multirow[t]{2}{*}{ Manihot esculenta Crantz ${ }^{(\mathrm{v})}$} & \multirow[t]{2}{*}{ Euphorbiaceae } & Feuille & 0,284 & 0,215 \\
\hline & & Tubercule & $0,252^{*}$ & 0,108 \\
\hline \multirow[t]{2}{*}{ Ocimum basilicum L. ${ }^{(\mathrm{v})}$} & \multirow[t]{2}{*}{ Lamiaceae } & Racine & $0,884^{\star *}$ & 0,213 \\
\hline & & Feuille & 0,113 & 0,422 \\
\hline \multirow[t]{2}{*}{ Parkia biglobosa (Jacq.) G. Don ${ }^{(\mathrm{v})}$} & \multirow[t]{2}{*}{ Fabaceae } & Ecorce & 0,154 & 0,084 \\
\hline & & Racine & 0,358 & 0,227 \\
\hline Pseudarthria hookeri Wight \& Arn. & Fabaceae & Ecorce & 0,298 & 0,213 \\
\hline Raphionacme brownii Scott-Elliot(v) & Apocynaceae & Tubercule & $0,509^{* *}$ & 0,070 \\
\hline Sarcocephalus latifolius (Sm.) E.A. Bruce ${ }^{(v)}$ & Rubiaceae & Ecorce & 0,344 & 0,224 \\
\hline \multirow[t]{2}{*}{ Sorghum bicolor (L.) Moench } & \multirow[t]{2}{*}{ Poaceae } & Tige & $0,426^{*}$ & 0,054 \\
\hline & & Panicule & 0,189 & 0,172 \\
\hline Tamarindus indica L. & Fabaceae & Feuille & 0,356 & 0,213 \\
\hline Terminalia avicennioides Guill. \& Perr. & Combretaceae & Feuille & 0,293 & 0,223 \\
\hline \multirow[t]{2}{*}{ Vernonia colorata (Willd.) Drake } & \multirow[t]{2}{*}{ Asteraceae } & Tige & $1,098^{* *}$ & 0,367 \\
\hline & & Feuille & 0,296 & 0,117 \\
\hline Vigna subterranea (L.) Verdc. & Fabaceae & Graines & 0,208 & 0,134 \\
\hline \multirow[t]{2}{*}{ Vigna unguiculata (L.) Walp. } & \multirow[t]{2}{*}{ Fabaceae } & Graines & $0,056^{*}$ & 0,025 \\
\hline & & Fanes & $0,198^{*}$ & 0,086 \\
\hline \multirow[t]{3}{*}{ Vitellaria paradoxa C.F. Gaertn. ${ }^{(v)}$} & \multirow[t]{3}{*}{ Sapotaceae } & Feuilles & $0,103^{*}$ & 0,049 \\
\hline & & Racine & $0,546^{*}$ & 0,218 \\
\hline & & Jeune feuilles & $0,466^{*}$ & 0,219 \\
\hline Nombre d'organes & & & $-0,043^{* *}$ & 0,006 \\
\hline
\end{tabular}

$(v)=$ représente les espèces vulnérables $;^{*}=$ significatif; ${ }^{* *}=$ hautement significatif; ${ }^{* * *}=$ très hautement significatif Coef = Coefficient de régression partielle; Coef.ET $=$ Erreur type des coefficients de régression * ${ }^{*}$ significant; ${ }^{* *}=$ highly significant; ${ }^{* *}=$ very highly significant Coef $=$ Partial regression coefficient; TE Coef $=$ Standard error of the regression coefficients 


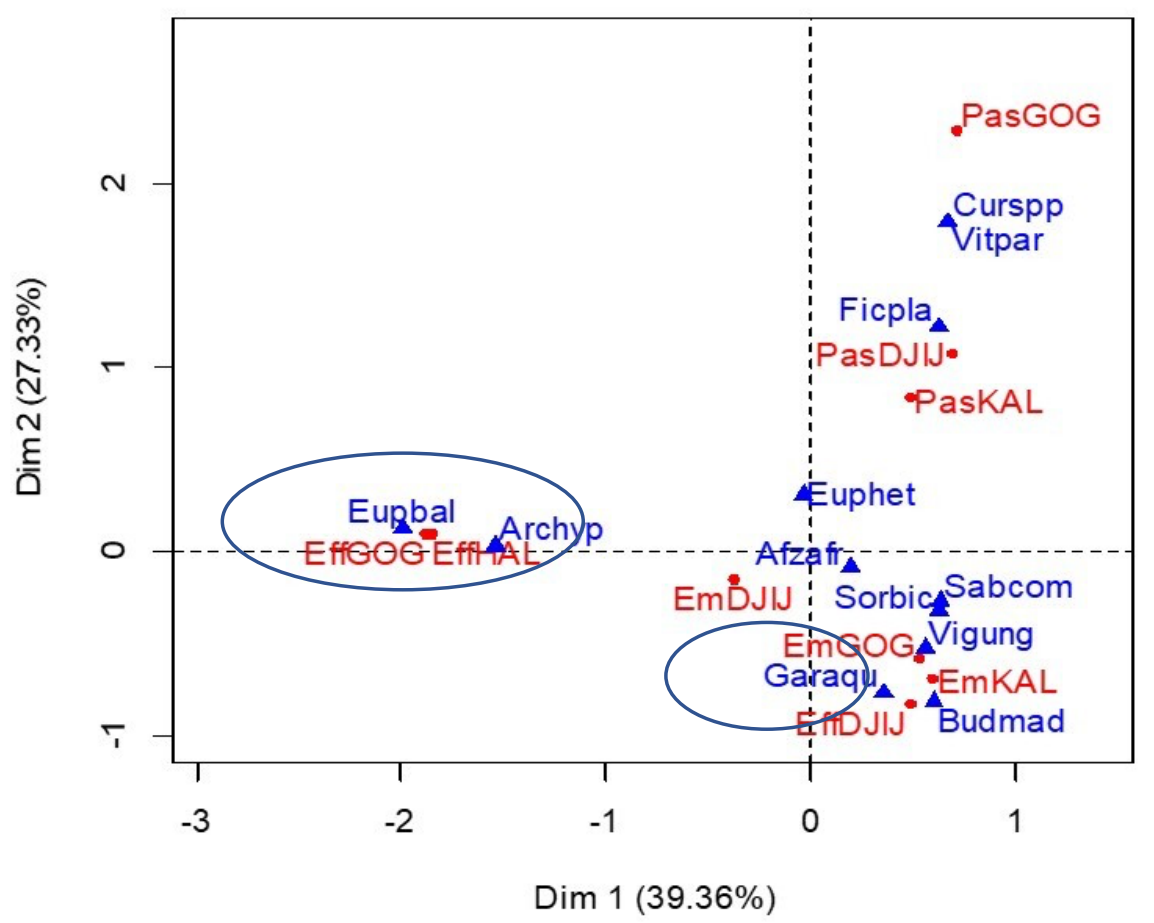

Figure 2. Relation entre les espèces végétales impliquées dans les recettes et la zone agro-écologique Figure 2. Relationship between the plant species involved in the recipes and the agro-ecological zone

Légende :

PasGOG: Efficacité passable

Gogounou

EmDJIJ: Efficacité moyenne Djidja

EffGOG: Efficacité Gogounou

Curspp: Curcubita spp

Euphet :Euphorbia heterophylla

Afzafr: Afzelia africana

Vigung: Vigna unguiculata

Legend :

PasGOG: Fair efficiency Gogounou

EmDJIJ: Average efficiency Djidja

EffGOG: Efficiency Gogounou

Curspp: Curcubita spp

Euphet: Euphorbia heterophylla

Afzafr: Afzelia africana

Vigung: Vigna unguiculata
PasDJIJ: Efficacité passable Djidja

EmGOG: Efficacité moyenne

Gogounou

EffLAL: Efficacité Kakalé

Vitpar: Vitellaria paradoxa

Eupbal: Euphorbia balsamifera

Sorbic: Sorghum bicolor

Garaqu: Gardenia aqualla

PasDJIJ: Fair effectiveness Djidja

EmGOG: Average efficiency

Gogounou

EffLAL: Kakalé efficiency

Vitpar: Vitellaria paradoxa

Eupbal: Euphorbia balsamifera

Sorbic: Sorghum bicolor

Garaqu: Gardenia aqualla
PasKAL: Efficacité passable Kalalé

EmKAL: Efficacité moyenne kalalé

EffDJIJ: Efficacité Djidja

Ficpla: Ficus platyphylla

Archyp: Arachis hypogaea

Sabcom: Saba comorensis

Budmad: Budgunnia

madagascariensis

PasKAL: Fair efficacy Kalalé

EmKAL: Average kalalé

efficiency

EffDJIJ: Effectiveness Djidja

Ficpla: Ficus platyphylla

Archyp: Arachis hypogaea

Sabcom: Saba comorensis

Budmad: Budgunnia

madagascariensis

\section{Forme et mode de préparation de la plante}

L'influence du mode de préparation des recettes sur la production laitière a été présentée dans le tableau 2. En dehors du nombre d'organes impliqués dans la constitution des recettes, le mode de préparation associé à chaque espèce végétale impactait la production laitière des vaches. Sur les treize espèces treize (13) qui ont eu d'effets significatifs avec la production laitière, les résultats du Tableau 2 renseignent que les modes d'utilisation se rangeaient successivement comme suit: Macération $>$ décoction > poudre > infusion ont été citées. Parmi 
les espèces qui ont fait objet de macération: Budgunnia madagascariensis (Desv.) J.H. Kirkbr. \& Wiersema, Arachis hypogaea L., Euphorbia balsamifera Aiton et Curcubita maxima L. ont été citées. Les espèces telles que: Gardenia aqualla Stapf \& Hutch, Sorghum bicolor (L.) Moench et Vittelaria paradoxa C.F. Gaertn ne pouvaient être efficaces que si l'utilisation se faisait par décoction. L'efficacité de Vigna unguiculata L. sur la production laitière ne pouvait être obtenue que par trituration pendant une heure. Quant à Saba comorensis, (Bojer ex A. DC.) Pichon le mode d'utilisation n'a été très efficace que par infusion $(P<0.001)$.

\section{Effet des additifs et de la voie d'administration sur la production du lait}

Aux préparations galactogènes, les agro-éleveurs ajoutaient des ingrédients tels que le lait de vache, la potasse, le sel, le sucre, le savon et même des produits d'animaux sauvages comme la graisse du varan (Varanus niloticus) et la peau d'hérisson (Erinaceus europaeus). En dehors du nombre d'organes et du mode de préparation associé à chaque espèce végétale, l'effet des additifs alimentaires tels que le sel et la potasse sont apparus significatifs $(P<0,05)$ sur la production laitière des vaches (tableau 2). L'addition de la potasse à la recette accroissait son efficacité $(P<$ 0,01). Par ailleurs, en tenant compte des voies d'administration des recettes, les résultats des travaux ont indiqué que l'administration des préparations par voie orale influençait positivement la production laitière $(P<0,05)$

\section{Discussion}

L'étude montre l'existence d'une forte corrélation entre l'âge des enquêtés, les espèces efficaces et la zone agroécologique. L'utilisation des préparations galactogènes de nos jours, semble se limiter aux adultes. Ce constat s'explique par le fait que la jeune génération ignore les vastes ressources médicinales disponibles dans son environnement au détriment des médicaments allopathiques disponibles, faciles à utiliser et offrant un soulagement immédiat. De plus, la jeune génération migre et ceux qui sont encore dans les zones reculées ne sont pas intéressés par l'élevage, ni les pratiques ancestrales de la médecine traditionnelle. De ce fait, les connaissances sur les pratiques ethno-vétérinaires et surtout sur les préparations galactogènes sont confrontées à de sérieux défis en raison de l'exode rural vers les villes à la recherche d'emplois. Toutefois, il est à signaler aussi que le manque de connaissances des jeunes sur l'utilisation des plantes galactogènes notamment, le moment de la collecte des plantes, la partie de la plante à utiliser, le mode de conservation et la formulation des recettes pour une meilleure efficacité pose d'énormes problèmes à cette génération dans l'adoption des pratiques endogènes pour améliorer la production laitière. Pour ces agro-éleveurs enquêtés, les connaissances ne sont pas transmises à tous les membres de la communauté telles quelles ont été héritées. Leurs craintes sont qu'elles soient mal utilisées par la jeune génération. Ce bénéfice du doute sur les aboutissants finaux des pratiques n'est pas à négliger car, chaque détenteur de savoir d'une lignée désigne un ou deux sages de la descendance à qui il confie les secrets. En dehors de cet aspect, pour les praticiens, la divulgation des connaissances endogènes peut aussi diminuer l'effets des préparations sur la production du lait (Idu et al., 2010; Ayenia et Kayodeb, 2019).

Contrairement aux espèces végétales telles que Arachis hypogaea, qui est une culture vivrière et Gardenia aqualla, une espèce ubiquiste, l'étude montre que Euphorbia balsamifera est cité comme espèce galactogène efficace dans l'amélioration de la production du lait. Il a été signalé par (Yossi et al., 2006) que cette espèce pousse sur les sols rocheux ou sablonneux de 0-800 m d'altitude dans des régions au climat tropical sec, ce qui justifie sa présence dans les zones montagneuses du NordBénin et son utilisation récurrente dans cette zone d'étude en comparaison avec le Sud Bénin où la plante est très mal connue. Divers auteurs (Déléké Koko, 2011; Akouedegni et al., 2012; Agbanou et al., 2018; Imorou et al., 2020) font également le même constat en ce qui concerne les études portées sur la diversité floristique des espèces végétales galactogènes utilisées au Bénin. Des 44 familles de plantes galactogènes inventoriées, il est à remarquer que 10 d'entre elles dont les Fabaceae, les Apocynaceae, les Euphorbiaceae, les Moraceae, les Meliaceae, les Poaceae, les Sapotaceae, les Asteraceae, les Lamiaceae et les Rubiaceae ont plus d'influence sur l'efficacité des recettes à stimuler ou à induire la production du lait chez les vaches. En se référant aux travaux antérieurs (Déléké Koko, 2011; Akouedegni et al., 2013; Cisse et al., 2016; Salifou et al., 2017; Maya, 2018; Zahoor et al., 2019), différentes familles de ces plantes contribuent à améliorer la production de lait chez les femelles. 
Tableau 2. Influence du mode de préparation sur la production du lait $\left(R^{2}=90,1 \%\right.$; Prob.Fisher $\left.(76 ; 466)<0,001\right)$ Table 2. Influence of preparation method on milk production $\left(R^{2}=90.1 \%\right.$; Prob.Fisher $\left.(76 ; 466)<0.001\right)$

\begin{tabular}{|c|c|c|c|c|}
\hline Espèces & Famille & $\begin{array}{l}\text { Mode de } \\
\text { préparation }\end{array}$ & Coef & Coef ET \\
\hline \multirow[t]{2}{*}{ Acacia sieberiana DC. } & \multirow[t]{2}{*}{ Fabaceae } & Décoction & 0,130 & 0,090 \\
\hline & & Poudre & $0,204^{*}$ & 0,086 \\
\hline \multirow[t]{3}{*}{ Afzelia africana Pers. } & \multirow[t]{3}{*}{ Fabaceae } & Macération & $-0,198$ & 0,131 \\
\hline & & Poudre & $0,121^{*}$ & 0,059 \\
\hline & & Trituration & $-0,311$ & 0,218 \\
\hline \multirow[t]{3}{*}{ Aganope stuhlmannii (Taub.) Adema } & \multirow[t]{3}{*}{ Fabaceae } & Décoction & $1,055^{\star *}$ & 0,274 \\
\hline & & Macération & $-0,956^{\star *}$ & 0,236 \\
\hline & & Poudre & $-0,798^{*}$ & 0,222 \\
\hline \multirow[t]{4}{*}{ Arachis hypogaea L. } & \multirow[t]{4}{*}{ Fabaceae } & Décoction & 0,171 & 0,113 \\
\hline & & Macération & $0,100^{* *}$ & 0,034 \\
\hline & & Infusion & 0,153 & 0,109 \\
\hline & & Macération & $0,201^{\star *}$ & 0,057 \\
\hline Burkea africana Hook (v) & Fabaceae & Macération & $-0,479$ & 0,267 \\
\hline \multirow[t]{2}{*}{ Gardenia aqualla Stapf \& Hutch } & \multirow[t]{2}{*}{ Rubiaceae } & Décoction & $0,696^{\star *}$ & 0,696 \\
\hline & & Macération & 0,251 & 0,145 \\
\hline \multirow[t]{2}{*}{ Cucurbita pepo L. } & \multirow[t]{2}{*}{ Cucurbitaceae } & Décoction & 0,081 & 0,044 \\
\hline & & Macération & $0,712^{\star *}$ & 0,013 \\
\hline \multirow[t]{2}{*}{ Euphorbia balsamifera Aiton } & \multirow[t]{2}{*}{ Myrtaceae } & Décoction & 0,073 & 0,039 \\
\hline & & Macération & $0,465^{*}$ & 0,214 \\
\hline Euphorbia convolvuloides Hochst. ex Benth. & Myrtaceae & Infusion & 0,298 & 0,213 \\
\hline Ficus glumosa Delile & Moraceae & Décoction & $-0,645^{\star *}$ & 0,187 \\
\hline \multirow[t]{2}{*}{ Ficus vallis-choudae Delile } & \multirow[t]{2}{*}{ Moraceae } & Décoction & $-0,630$ & 0,337 \\
\hline & & Macération & 0,399 & 0,238 \\
\hline Raphionacme brownii Scott-Elliot & Asclepiadaceae & Macération & 0,361 & 0,221 \\
\hline Saba comorensis (Bojer ex A. DC.) Pichon & Apocynaceae & Infusion & $1,112^{\star *}$ & 0,213 \\
\hline \multirow[t]{2}{*}{ Sorghum bicolor (L.) Moench } & \multirow[t]{2}{*}{ Poaceae } & Macération & 0,142 & 0,035 \\
\hline & & Décoction & $0,432^{* *}$ & 0,054 \\
\hline \multirow[t]{2}{*}{ Vitellaria paradoxa C.F. Gaertn. } & \multirow[t]{2}{*}{ Sapotaceae } & Macération & $-0,361$ & 0,221 \\
\hline & & Décoction & $0,211^{*}$ & 0,091 \\
\hline \multirow[t]{2}{*}{ Vigna unguiculata (L.) Walp. } & \multirow{2}{*}{ Fabaceae } & Macération & $-0,196$ & 0,113 \\
\hline & & Trituration & $0,412^{*}$ & 0,213 \\
\hline
\end{tabular}

${ }^{*}=$ significatif; ${ }^{* *}=$ hautement significatif; ${ }^{* * *}=$ très hautement significatif

Coef = Coefficient de régression partielle; Coef.ET= Erreur type des coefficients de régression * $=$ significant; ${ }^{*}=$ highly significant; ${ }^{* * *}=$ very highly significant Coef $=$ Partial regression coefficient; TE Coef $=$ Standard error of the regression coefficients 
Tableau 3. Influence des ingrédients et du mode d'administration sur la production laitière des vaches Table 3. Influence of ingredients and method of administration on milk production in cattle

\begin{tabular}{lll}
\hline Ingrédients & Coef & Coef.ET \\
\hline Lait Vache (Oui) & 0,321 & 0,214 \\
\hline Potasse & $\mathbf{0 , 2 9 3}^{\star *}$ & 0,223 \\
\hline Sel & $\mathbf{0 , 0 5 6 ^ { * }}$ & 0,025 \\
\hline Sucre & 0,196 & 0,113 \\
\hline Savon neutre & $-0,412$ & 0,213 \\
\hline Graisse Varanus niloticus & 0,344 & 0,224 \\
\hline Erinaceus europaeus & 0,162 & 0,095 \\
\hline Mode d'administration & & \\
\hline Voie transdermique & 0,165 & 0,092 \\
\hline Voie Orale (Oui) & $\mathbf{0 , 1 3 5 ^ { * * }}$ \\
\hline
\end{tabular}

${ }^{*}=$ significatif; ${ }^{* *}=$ hautement significatif; ${ }^{* *}=$ très hautement significatif

Coef = Coefficient de régression partielle; Coef.ET= Erreur type des coefficients de régression; * $=$ significant; ${ }^{*}$ $=$ highly significant; ${ }^{* *}=$ very highly significant, Coef $=$ Partial regression coefficient; TE Coef $=$ Standard error of the regression coefficients

Parmi les dix-neuf (19) espèces végétales appartenant aux dix (10) familles ayant plus d'influence sur la production du lait des vaches selon les agro-éleveurs, certaines ont déjà fait objet d'études approfondies. II en ressort que la capacité de ces espèces à stimuler la production de lait chez les vaches est due à certains métabolites secondaires tels que les alcaloïdes, les flavonoïdes, les polyphénols, les polyterpènes, les saponosides, les dérivés quinoniques, les stéroïdes, les terpènes, les hétérosides cardiotoniques et les tanins catéchiques qui peuvent induire la sécrétion de lait. (Oketch-Rabah et al., 1998Goyal et al., 2003; Déléké Koko, 2011; Akouedegni et al., 2012; Agani et al., 2019;), ont rapporté certains composés tels que les terpènes, les stéroïdes et les dérivés cardiotoniques qui caractérisaient les plantes galactogènes. Le criblage phytochimique de Euphorbia balsamifera montre que les feuilles, les tiges et les racines sont riches en stéroïdes, tanins, flavonoïdes, saponines et alcaloïdes (Kamba et Hassan, 2010). Les feuilles et la tige d'Arachis hypogaea contiennent des alcaloïdes, des saponines et des stéroïdes (Manekeng et al., 2018). La présence de saponines, de flavonoïdes et de tanins dans l'écorce de $B$. madagascariensis a également été mentionnée par (Chingwaru et al., 2019). Les résultats des études de (Obayomi et al., 2019; Olorunmaiye et al., 2019) ont montré que les graines d'Afzelia africana sont riches en alcaloïdes, tanins, saponines, flavonoïdes et terpénoïdes.

En ce qui concerne les espèces végétales telles que Gardenia aqualla, Calotropis procera, Sorghum bicolor, Saba comorensis, Vigna unguiculata, Ficus glumosa, Khaya senegalensis, Ocimum basilicum et Raphionacme brownii, les études récentes indiquent que ces espèces sont riches en alcaloïdes, flavonoïdes, polyphénols, triterpènes, saponosides, stéroïdes, terpènes, et tanins catéchiques. Par ailleurs, sur les 18 espèces végétales identifiées comme étant des plantes aux propriétées galactogènes très efficaces par cette étude, Gardenia aqualla, Calotropis procera, Sorghum bicolor, Vigna unguiculata, Ficus glumosa, Ocimum basilicum Raphionacme brownii, Arachis hypogaea, Euphorbia balsamifera, et Vitellaria paradoxa ont fait objet d'étude approfondie et les résultats ont révélé qu'elles améliorent la production en lait chez les mammifères y compris l'homme (Jose et al., 2008; Kamba et Hassan, 2010; Pandey, 2017; Aziz et al., 2018; Adejumo et al., 2019). Par contre, il en existe d'autres dont Budgunnia madagascariensis à haute potentialité galactogène qui n'ont pas encore fait objet d'études approfondies (Sani et al., 2019). Pour (Serunkuma et al., 2017), les thérapies alternatives utilisées en médecine ethno-vétérinaire méritent d'être promus car, les pathogènes bactériens responsables des mammites bovines deviennent pour la plupart résistants aux antibiothérapies conventionnelles mais sensibles aux activités antibactériennes des espèces végétales utilisées par les agro-éleveurs. Ainsi, le potentiel galactogène de ces plantes pourrait donc être soutenu par la présence de ces composés et l'effet thérapeutique des autres espèces accompagnatrices de la recette dans la préparation des solutions galactogènes.

En outre, les plantes galactogènes présentent certaines limites. L'efficacité de toutes les formules galactogènes adoptées par les agro-éleveurs traditionnels en relation avec les différentes thérapies n'est pas très documentée et vérifiée dans les laboratoires. Bien que certains travaux aient été réalisés dans différentes régions du monde, l'importance de l'utilisation des recettes galactogènes reste encore à développer. En raison de l'industrialisation et de l'urbanisation croissante, 
la superficie du couvert forestier a beaucoup régressé et par conséquent, la disponibilité de certaines espèces végétales impliquées dans les préparations des recettes sont devenues rares. C'est le cas de Raphionacme brownii, Budgunnia madasgariensis, Afzelia africana, Daniella oliverii et Vitellaria paradoxa. La plupart des espèces sus citées sont en extinction et aucun enregistrement authentique n'est fait, car les acteurs concernés ont acquis les savoirs de leurs ancêtres ou de leurs aînés (Hart et Bussmann, 2018).

Contrairement aux espèces Agonope stuhlmanii (écorce, feuille et tige), Vitellaria paradoxa (feuille jeune, racine) et Ficus glumosa (racine et écorce) où plus d'une des parties des plantes utilisées par les éleveurs ont une influence significative sur l'efficacité des recettes, les résultats montrent que toutes les parties d'une plante présumée galactogène n'ont pas les mêmes degrés d'efficacité sur la recette. Pour certaines espèces présumées galactogènes, c'est la feuille qui a plus d'effet (Afzelia africana, Euphorbia basalmifera, Vitellaria paradoxa et Kaya senegalensis). Pour d'autres, ce sont plutôt les graines (Arachis hypogea et Vigna unguiculata). Pour d'autres encore, ce sont les racines (Budgunnia madasgariensis, Saba comorensis, et Gardenia aqualla), les écorces ou les tiges (Daniella oliveri, Calotropis procera et Sorghum bicolor). II a été démontré que ces parties de plantes trouvent leur efficacité par la forte concentration en leur sein des substances nutritives telles que les minéraux en dehors de leur profil en composées phytochimiques (Ahmad et al., 2015; Disler et al., 2014; Khattak et al., 2015). Bien que l'utilisation de ces parties de plantes soit efficace dans la préparation pour améliorer la production du lait, elles constituent un véritable danger pour l'écosystème car, la plupart des espèces chez lesquelles ces différents organes sont utilisés ont déjà fait objet d'études, et ces travaux ont montré que les espèces telles que Afzelia africana, Budgunnia madagascariensis, Daniella oliveri, Saba comorensis Raphionacme brownii, et Kaya senegalensis sont considérées comme étant vulnérables (Dassou et al., 2014; Hussain et al., 2018). Par conséquent, l'utilisation abusive des parties comme la racine, l'écorce de ces plantes peuvent être fatale surtout qu'elles ne sont pas cultivées pour pérenniser la disponibilité des ressources. Alors, une base de données standard à cet égard doit être développée et acceptée par tous les acteurs concernés et des études approfondies doivent être prioritaires pour leur conservation car, l'utilisation préférée de ces espèces végétales présumées galactogènes constituent une menace à leur population par surexploitation.
Outre les parties ou organes de plantes impliquées dans la constitution des recettes, certains autres ingrédients étaient également incorporés aux préparations. C'est le cas de la potasse, du sel, du sucre, du lait, de la peau et de la graisse des animaux sauvages. Pourtant parmi ceux-ci, seules la potasse et le sel semblent avoir une influence significative sur la production du lait. Ce résultat peut s'expliquer par le fait, que le sel de par son goût, améliore la qualité organoleptique de la préparation dont l'éffet de consommation est l'amélioration de la production laitière chez la vache. Néanmoins, hormis l'appétence que procure le sel dans la recette, il joue un rôle antibactérien et participe à l'homéostasie cellulaire dans la répartition des liquides dans l'organisme des sujets traités (Schlienger, 2020). En ce qui concerne la potasse, son effet pourrait être dû à son activité énergétique par effet du propylène glycol; un précurseur du glucose permettant ainsi la synthèse et la libération du calcium qui joue un rôle primordial dans la synthèse des cellules galactophores et l'éjection du lait à travers les canaux galactopoétiques (I.N.R.A., 2013).

Par rapport aux méthodes de préparations des recettes, l'étude montre que la méthode par infusion, et décoction, et la macération ont plus d'influences significatives sur l'efficacité de la recette. Cela peut être expliqué par le fait que ces méthodes sont plus faciles à appliquer et moins coûteuses pour les populations qui l'adoptent. Cependant, elles permettent d'extraire le maximum de substances actives sur les cellules galactophores pour l'amélioration de la production du lait chez les vaches. Ces constats nous allient à ceux signalés dans divers travaux sur les plantes galactogènes (Ayoola et al., 2008; Kayode et al., 2015; Osabor et al., 2016). Ces recettes à base de plantes présumées galactogènes selon les agro-éleveurs, sont administrées par voie orale principalement à l'aide de flacons plastiques ou parfois à l'aide de calebasse artisanale. Cela se fait une seule fois en début de lactation et la préparation peut ne plus être utilisée jusqu'à la réforme des vaches. Ce mode d'administration des préparations est justifié par la présente étude comme étant le mode ayant un effet très significatif sur l'amélioration de la production du lait des vaches. Ce résultat s'explique par le fait que les agro-éleveurs n'ont pas d'autres alternatives. Ils sont limités par leur situation socio-économique et l'insuffisance de connaissances dans la transformation des préparations des produits injectables. Aussi, les produits sous forme injectable ne répondent plus à leur réalité. Ces produits de l'étranger ne respectent plus la tradition et les mythes liés à l'administration des préparations aux vaches par voie orale. 


\section{Conclusion}

L'étude permet de découvrir un rôle potentiel des espèces végétales impliquées dans la constitution des recettes galactogènes utilisées par les agroéleveurs du Bénin. Ces plantes peuvent constituer une stratégie alternative rentable dans la gestion des performances laitières des vaches laitières locales dans les zones agroécologiques du Bénin où l'élevage extensif des bovins constitue une activité principale. Toutefois, l'utilisation de certaines parties de ces espèces présumées galactogènes dans la formulation des recettes constituent un grand danger pour la préservation et la durabilité de l'écosystème. Ainsi, à défaut d'utiliser l'écorce et la tige de Aganope stuhlmanii, l'écorce de Daniellia oliveri, la tige de Calotropis procera, la plante entière de Euphorbia basalmifera, la racine de Ficus glumosa et la racine de vitellaria paradoxa, il est préférable d'opter pour les feuilles et les tiges de la plante. Par ailleurs, en ce qui concerne les espèces qui ne font intervenir que les racines pour être efficace dans les préparations, des études approfondies doivent être réalisées pour l'adoption probables d'autres parties telles que les feuilles et les tiges afin d'éviter leur destruction et leur disparition de la flore du Bénin. Néanmoins, l'utilisation de plantes médicinales dans le domaine de la recherche peut très probablement être encouragée et renforcée en lançant un programme coordonné de recherchedéveloppement pour évaluer et tester l'efficacité des plantes galactogènes utilisées en normalisant les méthodes de culture et de conservation de ces plantes. L'investigation sur les recettes galactogènes doit être intensifiée en tirant parti des progrès techniques. Par conséquent, le risque peut être réduit grâce à l'analyse d'effets secondaires à long terme inconnus ou insuffisamment connus. Toutefois, il est urgent que les mesures nécessaires soient prises pour conserver les espèces menacées telles que Budgunnia madasgariensis, Saba comorensis, Euphorbia basalmifera, Afzelia africana et Kaya senagalensis. Ces mesures doivent être aussi orientées vers les espèces galactogènes non encore identifiées comme étant vulnérables.

\section{Déclarations}

Ethiques d'approbation et consentement à participer: Toutes les personnes interviewées ont donné leur consentement préalable pour participer librement à l'enquête, en connaissant les objectifs exclusivement scientifiques de l'étude. Les auteurs soulignent que leur université ne possède pas de comité d'éthique en place pour la recherche ethnobotanique. Consentement à la publication: Tous les auteurs ont lu et approuvé la version finale du manuscrit avant soumission

Disponibilité des données et des matériaux: Les données collectées et traitées dans le cadre du présent travail sont disponibles uniquement auprès des auteurs.

Financement: Programme Fonds Compétitive de Recherche de l'Université d'Abomey-Calavi (PFCRUAC III) pour le financement du Projet.

Conflit d'intérêt: Les auteurs déclarent qu'ils n'ont aucun conflit d'intérêt.

Contributions des auteurs: La conception, la méthodologie, la validation et la collecte ont été réalisées sous le contrôle de BKC, ADA et ACG. Le traitement des données, la validation des résultats et la correction du document scientifique ont été réalisés par GAM. DJ est le coordonnateur du projet de financement et BS a supervisé la préparation du projet d'étude et la révision de la version finale du draft d'article. $A Z$ a réalisé l'enquête, traduit le résumé en anglais et rédigé le manuscrit jusqu'à sa version finale. Tous les auteurs ont lu, révisé et approuvé la version finale de l'article avant sa soumission.

\section{Références bibliographiques}

Aubertin C. 2018. Le protocole de Nagoya à l'épreuve de la recherche sur la biodiversité. In: Pomade A, éditeur. Hommes-Milieux: vers un croisement des savoirs pour une méthodologie de l'interdisciplinarité 99-111.

Adejumo AA, Ogunbiyi SV, Azeez GO, Olalubi AO. 2019. Laboratory qnalysis of Nigerian grown Ocimum gratissimum (Scent leaf) phytochemical constituents and antimicrobial activity for the control of bacteria infectious Diseases.

Agani Z, Akouedegni CG, Boko KC, Dossou J, Babatounde S. 2019. Modes de préparation et principes actifs des recettes galactogènes utilisées pour améliorer la productivité laitière des mammifères: Synthèse bibliographique 38-46.

Agbanou T, Abdoulaye D, Bogo GASO, Paegelow $M$, Tente B. 2018. Variabilité pluviométrique et son impact sur le couvert végétal dans le secteur Natitingou-Boukombé au nord-ouest du Bénin.

Ahmad K, Ahmad M, Weckerle C. 2015. Ethnoveterinary medicinal plant knowledge and practice among the tribal communities of Thakht-eSulaiman hills, west Pakistan. Journal of ethnopharmacology 170:275-83.

Akoègninou $A$, Van der Burg WJ, Van der Maesen LJG. 2006. Flore analytique du Bénin. Backhuys Publishers.

Akouedegni CG, Tossa IG, Daga FD, Koudandé DO. 2012. Synthèse des connaissances sur les plantes galactogènes et leurs usages en République du Bénin. Bulletin de la Recherche Agronomique 12. 
Akouedegni G, Delphin K, Edwige A, Sylvie A. 2013. Effects of leaves extract from Spondias mombin L. and Vitellaria paradoxa Gaertn F. on West African Dwarf (WAD) Sheep Performance in Republic of Benin. Journal of Animal Science Advances 3(2):74.

Ayeni EA, Basiri B. Ethnoveterinary survey of plants used in treating livestock among the Fulani people of Girei, Adamawa State, Nigeria. 2018. World News of Natural Sciences 16:53-66.

Ayenia MJ, Kayodeb J. 2019. Ethnobotanical Survey of Plants' Stem Barks Used in Kaduna State of Nigeria.

Ayoola GA, Coker HA, Adesegun SA, Adepoju-Bello AA, Obaweya K, Ezennia EC, Atangbayila TO. 2008. Phytochemical screening and antioxidant activities of some selected medicinal plants used for malaria therapy in Southwestern Nigeria. Tropical Journal of Pharmaceutical Research 7(3):1019-24.

Aziz MA, Khan AH, Adnan M, Ullah H. 2018. Traditional uses of medicinal plants used by Indigenous communities for veterinary practices at Bajaur Agency, Pakistan. Journal of ethnobiology and ethnomedicine 14(1):11.

Bullitta S, Re GA, Manunta MDI, Piluzza G. 2018. Traditional knowledge about plant, animal, and mineral-based remedies to treat cattle, pigs, horses, and other domestic animals in the Mediterranean island of Sardinia. Journal of ethnobiology and ethnomedicine 14(1):50.

Chingwaru C, Bagar T, Maroyi A, Kapewangolo PT, Chingwaru W.2019. Wound healing potential of selected Southern African medicinal plants: A review. Journal of Herbal Medicine 17:100263.

Cisse A, Gueye M, Ka A, Ndiaye F, Koma S, Akpo LE. 2016. Ethnobotanique des plantes médicinales chez les bergers peuls de Widou Thiengoly de la commune de Téssékéré (Ferlo-Nord Sénégal). Journal of Applied Biosciences 98:9301-8.

Dassou GH, Ouachinou JM-AS, Adomou AC, Yédomonhan $\mathrm{H}$, Tossou M, Favi A, Djidohokpin D, Gbèdolo $E$, Akoègninou A. 2020. Plant and natural product based homemade remedies for veterinary uses by the Peul community in Benin. Journal of Ethnopharmacology 261:113107.

Dassou HG, Ogni CA, Yédomonhan H, Adomou AC, Tossou M, Dougnon JT, Akoègninou A. 2014. Diversité, usages vétérinaires et vulnérabilité des plantes médicinales au Nord-Bénin. International Journal of Biological and Chemical Sciences 8(1):189-210.
DE. 2018. Annuaire Statistique du Ministère de l'Agriculture, de l'Elevage et de la pêche, Service Statistique, Cotonou, Bénin.

DE. 2019. Annuaire Statistique du Ministère de l'Agriculture, de l'Elevage et de la pêche, Service Statistique, Cotonou, Bénin.

Déléké Koko KIE. 2011. Exploitation traditionnelle des plantes galactogènes et emménagogues: ethnobotanique, efficacité thérapeutique, valeur d'usage et statut de conservation des principales plantes utilisées dans la Réserve de la Biosphère de la Pendjari (Bénin) [PhD Thesis]. Thèse de Doctorat Unique, Université d'Abomey-Calavi, Bénin.

Disler M, Ivemeyer S, Hamburger M, Vogl CR, Tesic A, Klarer F Meier B, Walkenhorst M. Ethnoveterinary herbal remedies used by farmers in four northeastern Swiss cantons (St. Gallen, Thurgau, Appenzell Innerrhoden and Appenzell Ausserrhoden). Journal of ethnobiology and ethnomedicine 10(1):32.

Dossou ME, Houessou GL, Lougbégnon OT, Tenté AHB, Codjia JTC. 2012. Etude ethnobotanique des ressources forestières ligneuses de la forêt marécageuse d'Agonvè et terroirs connexes au Bénin. Tropicultura 30(1).

Eynden V, Damme P. Medicinal and aromatic plants used by the topnaar - Namibia. 19933. Acta horticulturae (330):75-84.

Goyal RK, Singh J, Lal H. 2003. Asparagus racemosus-an update. Indian journal of medical sciences 57(9):408-14.

Hart R, Bussmann RW. 2018. Trans-Himalayan Transmission, or Convergence? Stantonia (Lardizabalaceae) as an Ethnoveterinary Medicine. Medicina nei Secoli 30(3):929-48.

Hussain W, Badshah L, Ullah M, Ali M, Ali A, Hussain F. 2018. Quantitative study of medicinal plants used by the communities residing in Koh-e-Safaid Range, northern Pakistani-Afghan borders. Journal of Ethnobiology and Ethnomedicine 14(1):1-18.

Idu M, Erhabor JO, Efijuemue HM. 2010. Documentation on medicinal plants sold in markets in Abeokuta, Nigeria. Tropical Journal of Pharmaceutical Research. 9(2).

Imorou L, Togbé EC, Hotegni NVF, Bello DO, Biaou BO, Nuer AT, Adoukonou-Sagbadja H, Ahoton LE. 2020. Galactogenic plant diversity, phenology and local in situ conservation practices in agro-ecological zones of Benin Republic. Genetic Resources and Crop Evolution 1-20. 
INRA. Inra Productions Animales. 2013 [Internet]. 2013. Disponible sur: https://www6.inrae.fr/productions-animales

Jose MA, Balasubramanian $S$, Rahman $A H$, Varghese NA, Kumar AS, Sumaiya A, Vivekanandhan S, Sridharan C, Radhakrishnan M. 2008. Diuretic activity of seeds of Cucurbita maxima Duchesne in albino wistar rats. indian journal of pharmacology p. 72-72.

Kamba AS, Hassan LG. 2010. Phytochemical screening and antimicrobial activities of Euphorbia balasamifera leaves stems and root against some pathogenic microorganisms. African Journal of Pharmaceutical Sciences and Pharmacy 1(1):57-64.

Kayode J, Omotoyinbo MA, Ayeni MJ, Oyedeji AA. 2015. Stem band roots extravitism in Ekiti State Nigeria: Need for conservation as a sustainable innovation in healthcare management in rural areas. American Journal of Bioscience. 3(2):28-33.

Khattak NS, Nouroz F, Rahman IU, Noreen S. 2015. Ethno veterinary uses of medicinal plants of district Karak, Pakistan. Journal of Ethnopharmacology 171:273-9.

Lesse P, Houinato MR, Djenontin J, Dossa H, Yabi B, Toko I. 2015. Transhumance en République du Bénin: états des lieux et contraintes. International Journal of Biological and Chemical Sciences 9(5):2668-81.

Manekeng HT, Mbaveng AT, Nguenang GS, Seukep JA, Wamba BEN, Nayim P, Yinkfu, NR, Fankam, AG, Kuete V. 2018. Anti-staphylococcal and antibiotic-potentiating activities of seven Cameroonian edible plants against resistant phenotypes 14.

Maya D. 2018. Ethnobotanical survey and biological activities of two lactogenic plants in the Cascades Region of Burkina Faso. Journal of Diseases and Medicinal Plants 4(1):1.

Mullah F, Kumar D, Antani D, Gupta M. 2013. Study of knowledge, perceptions and practices related to blood donation among the healthcare support staff of a tertiary care hospital in Gujarat, India. Online Journal of Health and Allied Sciences 12(1-2).

Obayomi MO, Suleiman B, Bashir AY. 2019. Proximate and anti-nutrient composition of Afzelia africana and Crescentia cujete seeds. Ife Journal of Agriculture 31(2):72-9.

Oketch-Rabah HA, Oduol E, Oluka MA, Nyamwaya D. 1998. Use of traditional and pharmaceutical medicines in Kenya: The case of Kisumu and Rachuonyo Districts in Luo Nyanza.
Olorunmaiye KS, Apeh LE, Madandola HA, Oguntoye MO. 2019. Proximate and phytochemical composition of African Mahogany (Afzelia africana) seed and African mesquite (Prosopis africana) pod. Journal of Applied Sciences and Environmental Management 23(2):249-52.

Osabor VN, Etiuma RA, Ntinya MU. 2016. Chemical profile of leaves and roots of miracle fruit (Synsepalum dulcificum). Chemical Science International Journal 1-8.

Pandey S. 2017. Phytochemical constituents, pharmacological and traditional uses of Ocimum gratissimum $\mathrm{L}$ in tropics. Indo American Journal of Pharmaceutical Sciences 4(11):4234-42.

R Core Team. 2019. R: a language and environment for statistical computing. Vienna (Austria): The publisher is R Foundation for Statistical Computing.

Salgotra RK, Zargar SM, Sharma M, Sood M. Traditional Knowledge. 2018. A therapeutic potential in the scenario of climate change for sustainable development. Development 61(1-4):140-8.

Salifou CFA, Kassa KS, Ahounou SG, Moussa H, Dotché IO, Agbozo JM, Issifou MT, Youssao IAK. 2017. Plantes lactogènes des bovins et leurs modes de préparation dans les élevages traditionnels au Bénin. Livestock Research for Rural Development 29(2):29.

Sani NA, Kawu MU, Bako IG. 2019. Effects of Launaea taraxacifolia and resveratrol on milk yield and serum prolactin and oxytocin levels: a lactogenic study. International Journal of Veterinary Science and Medicine 1-7.

Schlienger JL. 2020. Une histoire qui ne manque pas de sel! Médecine Mal Métaboliques.

Sébastien L, Josse J, Husson F. 2008. FactoMineR: an $R$ package for multivariate analysis. Journal of statistical software. 25(1):1-18.

Serunkuma P, Mcgaw LJ, Nsahlai IV, Van Staden J. 2017. Selected southern African medicinal plants with low cytotoxicity and good activity against bovine mastitis pathogens. South African journal of botany 111:242.

ul Haq Z, Syed MS, Khan SM, ur Rahman A. 2019. Ethnoveterinary practices used for the treatment of different ailments in District Swabi, Khyber Pakhtunkhwa, Pakistan.

Yossi H, Kaya B, Traoré CO, Niang A, Butare I, Levasseur V, Sanogo D. 2006. Les haies vives au Sahel: état des connaissances et recommandations pour la recherche et le développement. World Agroforestry Centre. 


\section{Annexe}

Questionnaire № 1 : Inventaires des recettes ethnovétérinaires utilisées par les agroéleveurs pour améliorer la production laitière des vaches locales de race Borgou des plantes galactogènes par les éleveurs destiné pour le focus group

I. IDENTIFICATION ET LOCALISATION DE LA ZONE du Focus group

1. $\mathrm{N}^{\circ}$ de la fiche:

2. Coordonnées GPS :

3. Nom et Prénoms de l'enquêteur :

4. Date de l'enquête : /........./20... /

5. Département :...................... (1= Borgou; 2 = Alibori; $3==$ Zou $)$

6. Commune................. (1= Kalalé ;2= Djidja; 3 = Gogounou $)$

7. Arrondissement :

8. Quartier/Village/Hameau/Campement/Transhumant :

\section{CARACTERISTIQUES SOCIO-DEMOGRAPHIQUE DE L'ENQUETE}

1. Nombre d'enquêtés :

2. Sexe :

(0= Féminin; $1=$ Masculin $)$

3. Age des répondants (Age révolu) :

4. Groupe sociolinguistiques de l'enquêté :

1= Peulh; 2= Bariba; 3= Gando; 4= Dendi

5. Avez-vous reçu une éducation formelle (Instruction)?.

(1= Oui, $0=$ Non $)$

6. Si Oui, quelle est le niveau atteint (Niveau d'instruction) :

1= Primaire; 2 = Secondaire (préciser la classe); 3 = Formation technique;

7. Etes-vous alphabétisés?

8. Si Oui, que savez-vous faire ?.

Compter; $5=$ Lire, écrire et compter)

9. Situation matrimoniale des l'enquêtés?

10. Religion de l'enquêté ?.

(1= lire; $2=$ écrire; $3=$ lire et écrire; $4=$

11. Origine des enquêtés ?

1= Autochtone du village; 2 = en transhumance saisonnière; 4= autre (à préciser)

12. Si l'enquêté n'est pas autochtone, depuis combien d'années réside-t-il dans le village ?...

13. Activité principale de l'enquêté :

1= éleveur; $2=$ Producteur de lait; $3=$ Producteur de fromage; $4=$ agriculteur; $5=$ transformation de produits agricoles,

\section{USAGE DES PLANTES GALACTOGENES DANS LA PRODUCTION DE LAIT}

14. Reconnaissez-vous ces plantes suscitées? (1= Oui, $0=$ Non $)$

15. Si Oui quel rôle jouent t-elles ?

16. Utilisez-vous ces plantes pour améliorer la production de lait des vaches? $(1=$ Oui, $0=$ Non $)$

17. Si oui comment?

18. Quelles sont les organes les plus efficaces?

19. Depuis quand utilisez-vous ces plantes pour aider les vaches à mieux produire le lait des vaches ? 
20. Combien de plantes galactogènes combinez-vous pour améliorer la production laitière des vaches ?

21. Citez-les.

22. Si vous devez allouer des scores; quel score serait-il pour chacune des plantes suivantes ?

\begin{tabular}{|l|l|l|l|l|}
\hline Plantes & & & & \\
\hline Budgunnia madascariensis Cocobit & & & & \\
\hline Saba comorensis ( Bernine) & & & & \\
\hline Tcholouwi (Euphorbia basalmifera) & & & & \\
\hline Kaya senegalense (Kahi & & & & \\
\hline Dignaali (Gardenia erubescens) & & & & \\
\hline In-indè ( Euphorbia hetérophylla) & & & & \\
\hline Parkia biglosa ( karité) & & & & \\
\hline Adansonia digitata L. & & & & \\
\hline Afzelia africana Pers. & & & & \\
\hline Aganope stuhlmannii (Taub.) Adema & & & & \\
\hline Arachis hypogaea L. & & & & \\
\hline Balanites aegyptiaca (L.) Delile & & & & \\
\hline Carica papaya L. & & & & \\
\hline Daniellia oliveri (Rolfe) Clapier. \& Dalziel & & & & \\
\hline Calotropis procera subsp. hamiltonii (Wight) Ali & & & & \\
\hline Ficus glumosa Delile & & & & \\
\hline Ficus vallis-choudae Delile & & & \\
\hline Manihot esculenta Crantz & & & & \\
\hline Ocimum basilicum L. & & & \\
\hline Vigna unguiculata L. & & & \\
\hline
\end{tabular}

23. En quoi ces plantes sont-elles meilleures ?

1= amélioration en lait; 2= développement des mamelles; 3= bonne conformation; 4= amélioration du gout par le veau; $5=$ appétit de la vache;

24. Faites-vous la combinaison d'une de ces plantes avec d'autre(s) ingrédients?

25. Si oui le(s)quelle(s)? $(1=$ Oui, $0=$ Non $)$ (n)

26. Sous quelle forme préférez-vous utiliser la plante ? Après traitement ;)

27. Si desséché, méthode de séchage : 1= Fraiche; $2=$ Desséché; $3=$ 4= Autre (à préciser) :

28. Si après traitement, précisez le traitement

29. Quel est le mode de préparation galactogène préférez vous ? .............................. $1=$ Infusion; $2=$ Décoction; 4= trituration par breuvage; $6=$ poudre à lécher; $7=$ macération $8=$ extraction à froid

30. Quel est le mode d'administration de la préparation galactogène ? $1=$ Oral; 2= Massage des pis ;

31. Quelle quantité (en $L$ ) de la préparation donnez-vous à une vache par jour ?

32. Avec quelle fréquence donnez-vous la préparation à la vache?

33. Pouvez-vous nous dire en quelques mots comment vous préparez et administrez les préparations à base de plantes galactogènes aux animaux? 
IV. IMPACT DE L'UTILISATION DES PLANTES GALACTOGENE SUR LES ANIMAUX

1. Quelle quantité de lait produisez-vous par jour avant l'utilisation des plantes galactogènes ?

2. Quelle quantité de lait produisez-vous par jour après l'utilisation des plantes galactogènes ?

3. Conservez-vous le lait quelque temps après sa production ? ............................. 1= Oui, $0=$ Non

4. Si oui, la durée de conservation du lait obtenu grâce aux plantes galactogènes a-t-elle augmentée ? $1=$ Oui, $0=$ Non

5. Si oui, quelle est la variation de la durée de conservation entre le lait ordinaire et le lait obtenu grâce aux plantes galactogènes ?

6. Avez-vous remarqué un impact de l'utilisation des plantes galactogènes sur la santé des vaches ou des veaux ? $1=$ Oui, $0=$ Non

7. Si oui, les-quelles ? ........................................ 1 = diarrhée; $2=$ constipation; $3=$ Autres 